\title{
Factors determining choice of delivery place among women of child bearing age in Dega Damot District, North West of Ethiopia: a community based cross- sectional study
}

\author{
AlemayehuSayih Belay ${ }^{1 *}$ and EndalewGemechu Sendo ${ }^{2}$
}

\begin{abstract}
Background: In the latest report of Ethiopia Demography and Health Survey (EDHS) 2011, the Maternal Mortality Ratio was estimated at 676/100,000 live births. Most of these deaths are preventable. Increasing the proportion of women who deliver in a health facility can be an important means in reducing maternal mortality in low-income settings including Ethiopia.

We aimed to identify factors determining choice of delivery place among child bearing age women.

Method: A community based cross sectional survey was conducted in Dega Damot District from April- May, 2014. Mixed methods were employed in the study. Multistage sampling method was used. The primary outcome variable for this study was women who delivered their most recent baby in a health facility.

Result: Three hundred sixty one women who gave birth in the past 1 year were included in the study. The mean age of the respondents was 30.9 [SD \pm 6.006 ]. One hundred seven $(29.6 \%)$ of the respondents were in the age range of 25-29 years. In our study, the proportion of women assisted by skilled health workers during institutional delivery was $89.1 \%$ followed by Health extension workers (8.0 \%). Most women $(87.4 \%)$ who did not deliver in health facilities were assisted by families, friends or neighbors followed by Health extension workers (7.2 \%), and traditional birth attendants (5.4\%), respectively.

The qualitative data has described and gave an insight of the contributing factors that influence the women using the health institutions for delivery. These included: ANC attendance, Positive attitude of Health workers and complications during labor and delivery. The preference for a health facility delivery was largely due to the understanding that if complications occurred either during labor or delivery, this was the only place where they could be managed.
\end{abstract}

Conclusion: The study revealed that women's institutional delivery service utilization in the study area is low. Based on these findings, improving the utilization of health facility for delivery through educating women and health promotion have been recommended. This would help reduce the complications and dangers that often characterized home-based, unsupervised delivery.

Keywords: Institutional delivery, Women of reproductive age, Dega Damot District, Ethiopia

\footnotetext{
* Correspondence: Alex.sayihalem2012@gmail.com

1 Department of Nursing, Mizan Tepi University, College of Health Sciences,

Mizan Teferi, Ethiopia

Full list of author information is available at the end of the article
} 
(Continued from previous page)

Abbreviations: ANC, Antenatal Care; CSA, Central Statistical Agency; EDHS, Ethiopian Demographic and Health Survey; FGD, Focused Group Discussion; HCW, Health Care Workers; HEW, Health Extension Workers; IRB, Institutional Review Board; MDG, Millennium Development Goals; MMR, Maternal Mortality Ratio

\section{Background}

Millennium Development Goal 5 (MDG 5) set a target of $75 \%$ reduction in maternal mortality, from 400/ 100,000 live births to $100 / 100,000$ between the 1990 baseline and 2015 [1]. Increasing the proportion of women who deliver in a health facility can be an important means in reducing maternal mortality in low-income settings. It is globally recognized that one of the main challenges to achieving the MDG 5 of a global reduction of maternal death by $75 \%$ by 2015 is the low proportion of women who deliver with a skilled birth attendant [2].

In many low-income settings with a high burden of maternal deaths, few women use facilities for birth, often choosing a higher-risk birth at home, often without professional medical assistance [3]. Deliveries in health facilities can ensure that women are attended by skilled personnel and also link women to the referral system in the case of any complications.

A key strategy for reducing maternal morbidity and mortality is thus ensuring that every birth occurs with the assistance of skilled health personnel, meaning a medical doctor, nurse or midwife. Progress in raising the proportion of births delivered with skilled attendance has been modest over the course of the MDG time frame, reflecting lack of universal access to care [4].

According to Mini Ethiopian Health Demography Survey (MEDHS) 2014, $40 \%$ of pregnant women who gave birth in the 5 years preceding the survey received antenatal care once from a skilled provider, that is, from a doctor, nurse, or midwife, for their most recent birth, $34 \%$ from a nurse or midwife, and $6 \%$ from a doctor. But only $32 \%$ of women with a live birth in the 5 years before the survey made four or more ANC visits during the length of their pregnancy, a marked improvement from $19 \%$ reported in the 2011 EDHS [5].

Nevertheless, only $15 \%$ of births in Ethiopia are delivered at a health facility-14 \% in a public facility and $1 \%$ in a private facility [5].

As a signatory of Millennium Declaration 2000, Ethiopia is dedicated to improving skilled birth care and hence reducing maternal mortality. It targets for $75 \%$ reduction of MMR from 880/100,000 in 1990 to 220/ 100,000 in 2015. However, Ethiopia is still far off from the MDG 5 [6]. Ethiopia was also the least achiever in at least 4 visits antenatal care coverage in Sub-Saharan Africa. To be more objective, the proportion of health facility delivery and antenatal care between 1995 and 2011 were in the range of $5-15 \%$ and $10-19 \%$, respectively [5-7]. Thus, the low proportion of antenatal care compounded by the extremely low skilled person attended delivery might be some of the major reasons for the high maternal mortality persisting during the last decade (873 and 676 per 100,000 live births in 2000 and 2011, respectively) [7]. Our present study focused on West Gojjam Zone because its utilization of SBA services is lower compared to other regions of Ethiopia.

\section{Methods}

\section{Study design and study setting}

A community based cross-sectional study mixed methods was conducted in Daga Damot District from April 2014 to May 2014.

Daga Damot District is located $399 \mathrm{Km}$ from the capital city of Addis Ababa. It is one of the Districts in the West Gojjam, Amhara Region, Ethiopia. It has an area of $65726.68 \mathrm{Km}^{2}$ with an estimated total population of 152,343 according to the 2007 national census which was conducted by the Central Statistical Agency of Ethiopia (CSA). The estimated total number of women of reproductive age group and pregnant women in the district (both rural and urban) were 70,639 and 6699, respectively. At present, the district has 30 rural and 1 urban kebeles (the smallest administrative units in urban and rural areas). A total of 6 health centers and 31 Health posts were available in the District [8].

\section{Sample size determination and sampling procedures}

A single population proportion formula, $[n=(Z \alpha / 2) 2 p$ $(1-p) / d 2]$, was used to estimate the sample size. The following assumptions were made while calculating the sample size. The degree of precision or margin of error chosen to be 0.05 with the reliability coefficient of $1.96 \%$ certainly $(\mathrm{z}=1.96)$. The proportion of deliveries conducted in West Gojjam Zone, Sekela District where $(12.1 \%)$ of them gave birth at health facilities while majority of them $(87.9 \%)$ delivered at home [9]. Therefore, the proportion of delivery attended by skilled birth attendants was $12.1 \%(p=0.121$ and $q=0.879)$. As multistage sampling technique was used, we multiplied the calculated sample size by a design effect of 2 and added $10 \%$ for non-response. Then the total sample size calculated was 361.

Multistage sampling method was used to select 361 women of child bearing age (15-49 years). In Dega Damot District, there are 31 kebeles (30 rural kebeles and one urban kebele). Among 31 kebeles, one urban 
and eight rural kebeles were selected by simple random sampling technique. The total sample size was then allocated proportionately to the number of designated kebeles. Women of child bearing age (15-49 years) who gave birth within the past 2 years and lived in Dega Damot District for a minimum of 1 year prior to the survey were included in the study. When there were more than one eligible women in the selected household, a lottery method was used to pick one of them.

\section{Data collection tools and procedure Quantitative data}

Data were collected by using a pretested and structured questionnaire administered by face to face interviews. The questionnaire was adapted from other similar studies (9-11). The questionnaire was originally developed in English; then translated into local language (Amharic) and back into English to ensure its accuracy by an expert. To ensure the quality of data, the questionnaire was pretested in $5 \%$ of the sample size in similar set up before the actual data collection. The questionnaire consists of socio-demographic characteristics (age, ethnicity, religion, educational status and occupational status and obstetric history including women's place of delivery for their last child birth, women's past obstetrical history and others. Data were collected by trained 6 female health extension workers (HEW's) who were working in Health posts.

\section{Qualitative data}

This study was also triangulated with a qualitative method. Semi-structured interview guide was used to collect the data. Three focus group discussions (FGDs) were conducted each involving a total of 6-8 participants. Out of the three FGDs, one was conducted in Urban district while the remaining two FGDs were conducted in rural districts. Each group consisted of women within the age range of 18-45 years who delivered at home in the past 12 months and reported for $\mathrm{MCH}$ clinic registration or postpartum care. The women were selected purposively in the clinics and were followed into the communities where the health facilities are located and the FGDs were held. Permission to participate in the focus group was sought prior to the meeting. The data collection team included the researcher and two female research assistants. The female research assistants were hired as data collectors in order to guarantee the clients' openness to discussions and to conform to the study's feminist Theoretical stances. The discussions were audio-taped using a digital voice recorder and a trained data collector took written notes. Each session lasted for 30-40 min.

The discussions were focused on different themes relating to factors preventing utilisation of health facilities for delivery care including the women's previous experience with health facilities for labour and delivery care; attitude of health workers; perceived ANC care; and their choice of delivery place.

\section{Data analysis}

Data were cleaned and entered into EPI INFO version 3.5.4 and were exported to SPSS version 20.0 for data analysis. The data were expressed in percentages, graphs, means and standard deviations.

The qualitative data analysis was done simultaneously with data collection. Thematic analysis was used and all data were transcribed verbatim. All FGDs were transcribed and translated into English by two investigators fluent in Amharic. Where there was no consensus, the investigators reviewed the transcriptions and original recordings until agreement was reached. The transcripts were then categorized into themes and analyzed manually. All data were stored in a lockable cupboard and were only accessible to the researchers.

\section{Results \\ Socio-demographic characteristics}

A total of 361 eligible women were included in the study yielding the response rate of $100 \%$. The mean and median age of the respondents was 30.9 and 30.0 respectively. One hundred seven $(29.6 \%)$ of the respondents were in the age range of 25-29 years. Majority 296 (82\%) were rural residents. As regards their marital status, about $287(79.5 \%)$ of them were married. Among the total study participants, majority 360 (99.7 \%) were orthodox religion and all of them were Amhara by ethnicity. Two hundred fifteen $(59.6 \%)$ of them were farmers followed by house wives, $44(12.2 \%)$. Of the total respondents, more than half, $182(50.4 \%)$ were illiterate while $38(10.5 \%)$ of them had diploma and above. The mean monthly income of respondent's was \$ 41.6 USD [SD $\pm \$ 30.94$ USD] (Table 1).

\section{Institutional delivery use and attendants during delivery} In this study, of the total respondents only $38.2 \%$ of women gave birth in health facilities for their most recent birth while the most $223(61.8 \%)$ delivered at home. Home delivery in our study is defined as a delivery that is not being attended by a trained health worker using a safe delivery kit or attended by non-trained people (the majority of whom are family members) during delivery. In our study, the proportion of women assisted by skilled birth attendants during institutional delivery was $89.1 \%$ followed by Health extension workers (8.0 \%). Skilled birth attendant is an accredited health professional such as a midwife, doctor or nurse - who has been educated and trained to proficiency in the skills needed to manage normal (uncomplicated) pregnancies, 
Table 1 Socio-demographic characteristics of the respondents $(n=361)$ in Dega Damot District, Ethiopia, May 2014

\begin{tabular}{lll}
\hline Variables & Frequency & Percent \\
\hline Place of residence & & \\
Rural & 296 & 82.0 \\
Urban & 65 & 18.0
\end{tabular}

Age of respondents

$\begin{array}{ll}20-24 & 55 \\ 25-29 & 107 \\ 30-34 & 89 \\ 35-39 & 71 \\ +40 & 39 \\ \text { Mean + SD } & 30.93 \pm 6.006\end{array}$

Marital status

$\begin{array}{ll}\text { Married } & 287 \\ \text { Single } & 7 \\ \text { Divorced } & 11 \\ \text { Separated } & 39 \\ \text { Widowed } & 17 \\ \text { Religion } & \end{array}$

Orthodox

Protestant

Ethnicity

Amhara

Respondent's occupation

House wife

Governmental Worker

Merchant

Farmer

Daily labors

Student

Husband's occupation, $n=326$

Farmer

287

Daily laborer

Merchant

Governmental Worker

Respondent's educational status

Illiterate
Read and write
Primary education (1-8)
Secondary education
Certificate
Diploma and above

Husband's educational status, $n=326$

Illiterate

Read and writes
Table 1 Socio-demographic characteristics of the respondents $(n=361)$ in Dega Damot District, Ethiopia, May 2014 (Continued)

\begin{tabular}{lll}
\hline Primary education (1-8) & 50 & 15.3 \\
Secondary education & 16 & 4.9 \\
Certificate & 16 & 4.9 \\
Diploma and above & 57 & 17.5 \\
Monthly house hold income (USD) & & \\
$\$<=19.95$ & 77 & 21.3 \\
$\$ 19.95-34.95$ & 102 & 28.3 \\
$\$ 35-49.95$ & 78 & 21.6 \\
$\$>=50$ & 104 & 28.8 \\
Mean \pm SD & $41.6 \pm 619.5$ & \\
\hline
\end{tabular}

childbirth and the immediate postnatal period, and in the identification, management and referral of complications in women and newborns.

Most women $(87.4 \%)$ who did not deliver in health facilities were assisted by Lay birth attendants (families, friends or neighbors) followed by Health extension workers (7.2\%), and traditional birth attendants (5.4\%), respectively. A Lay birth attendant is a person without any training in midwifery skills who assisted a mother to give birth without delivery kit (Table 2).

\section{Reasons for utilization of institutional delivery}

Women who delivered in health facilities were asked the reasons for doing so. Among the respondents who attended institutional delivery, the main reasons cited for selecting skilled delivery service were multiple and included fear of complications (77.5\%), to get better service $(56.5 \%)$ safe and clean delivery $(46.4 \%)$ and they were communicated to deliver at health facilities during ANC attendance, 47 (35.5 \%) (Fig. 1).

Table 2 Percentage distribution of women who delivered in the past 1 year according to place of delivery and attendants during delivery, Dega Damot District, East Gojjam 2014

\begin{tabular}{lll}
\hline Variable & Number $(n=361)$ & Percent (100 \%) \\
\hline Place of delivery & & \\
Home & 223 & 61.8 \\
Health facility(HF) & 138 & 38.2 \\
Attendant during HF delivery & & \\
Health workers & 123 & 89.1 \\
Health Extension workers & 11 & 8.0 \\
Don't remember & 4 & 2.9 \\
Attendant during home delivery & & \\
Family/friends/neighbors & 195 & 87.4 \\
Health Extension workers & 16 & 7.2 \\
Traditional birth attendant & 12 & 5.4 \\
\hline
\end{tabular}




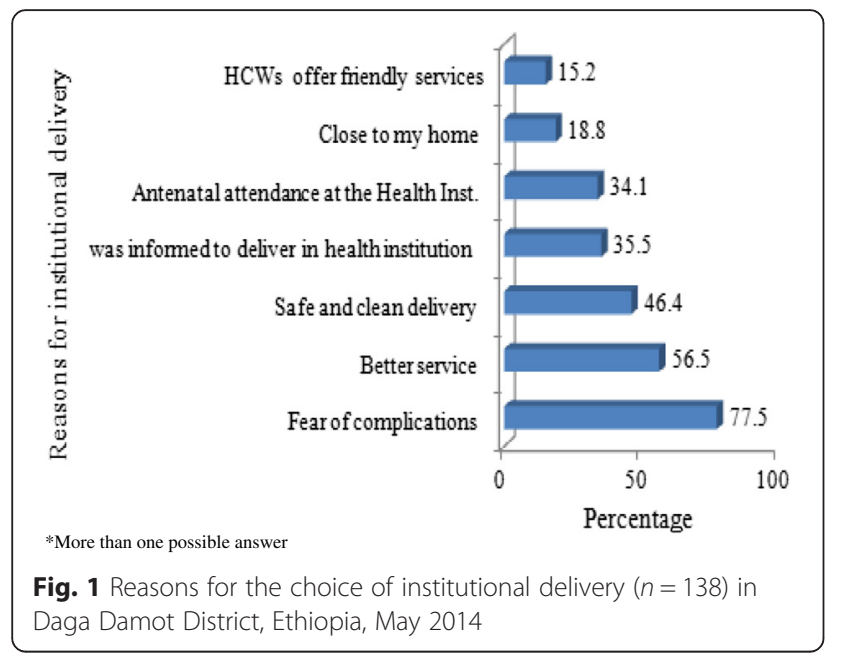

The qualitative data has supported the quantitative findings. One of the FGD participants has said: "Benefits of ANC are well- recognized in our community. For example, pregnant women who had a good knowledge and attended ANC clinic usually delivers at health institution. In addition, women attend health institution for delivery after they encountered complications such as too much bleeding and/or prolonged labor". (A 35- year old FGD participant)

\section{Past obstetric characteristics}

Two hundred and seven (57.3\%) respondents reported that they were less than 18 years old during their first pregnancy. One hundred thirty five $(37.4 \%)$ had attended ANC service, of which 59 (43.7 \%) had attended 2-4 visits, 41 (30.4\%) had attended one visit and 35 (25.9 \%) had more than four visit during their last pregnancy (Table 3).

Health care provider's behavior and attitudes are also determinant factor for a choice of place of delivery for pregnant women, some of the health workers are impolite, with offensive language and refuting to support the patients, these attitudes inhibit the women to deliver in health facilities, and nevertheless, positive attitudes of health workers draw women to deliver in health facilities. For example in the present study one woman during focused group discussion said:

"During my last few days before I delivered, I went to the ANC clinic, and the midwife advised me sympathetically that I had to go to the district hospital for delivery because my child was not in the right position. I went to the district hospital and gave birth to healthy baby". (FGD participant, aged 32 years).

Another experience from FGD with a mother who had given birth a month ago, gave a clear picture of a
Table 3 Obstetric and maternal characteristics $(n=361)$ in Dega Damot District, Ethiopia, May 2014

\begin{tabular}{lll}
\hline Variables & Frequency & Percent \\
\hline $\begin{array}{l}\text { Age at first marriage } \\
\quad 18 \text { years. }\end{array}$ & 207 & 57.3 \\
$>18$ years. & 154 & 42.7 \\
Age at first pregnancy & & \\
$\quad<18$ years. & 91 & 25.2 \\
$>18$ years. & 270 & 74.8 \\
Gravidity & & \\
1 & 99 & 27.4 \\
$2-5$ & 202 & 56.0 \\
$>5$ & 60 & 16.6 \\
Parity & & 42.9 \\
1 & 155 & 51.5 \\
$2-5$ & 186 & 5.5 \\
$>5$ & 20 & \\
Antenatal care attendance & & 37.4 \\
Yes & 135 & 62.6 \\
No & 226 & \\
No. of visits of antenatal care & & 30.4 \\
1 & 41 & \\
$2-4$ & 59 & \\
$>4$ & 35 & \\
\hline
\end{tabular}

provider-client relationship. "When I went to the health facility for delivery, I was impressed by the nurse who cared for me so much. She was so human, respectful and compassionate" (FGD Participant, aged 28 years).

Vaginal examinations women are exposed to in labor and delivery is frequently regarded and stated as painful, recurrent and regularly quoted as a factor preventing the use of health facility for delivery. In the current study one participant shared her lived experience as stated beneath:

"In my previous delivery I was exposed to series of vaginal examination....... Had it been my first delivery with the health facility, then I would not ever go there again". My faith (Muslim) doesn't let another person to insert his hands into a married woman's genital parts (Vagina). (FGD Participant, 34 years).

\section{Discussion}

In Ethiopia, just like most developing countries, a number of women still prefer to deliver at home than to deliver in the health facilities. In Ethiopia, only $15 \%$ percent of births are delivered at a health facility despite more than $40 \%$ of pregnant women having at least one ANC visit during pregnancy. In this study, we found 
only $38.2 \%$ of women gave birth in health facilities for their most recent birth. This prevalence is lower than the prevalence in studies conducted in Uganda and India $[10,11]$. Nevertheless it is relatively higher compared to the national average (15\%) reported in mini Ethiopian DHS of 2014. Consequently, additional efforts are required to warrant that the Ethiopian national target of $62 \%$ skilled delivery service is realized [12].

Several previous quantitative studies revealed that women's use of health facility delivery services is influenced by their demographic background characteristics and their socioeconomic status. A study in rural India showed that institutional delivery is much more common for first births than for subsequent births [13]. Concerning age at delivery, another study in rural India, Punjab, revealed that institutional deliveries were more common in comparatively younger age groups, at $43 \%$ for women age 18-25 compared with $23 \%$ for women age 36-45 [14]. However, in our study age of the women and parity did not show any significant association with the use of health facility delivery services.

In this study, some significant factors that were found to be associated with institutional delivery were levels of education, average monthly family income, and ANC attendance. Higher levels of education and optimally attending ANC services surge the likelihood of delivering in a health facility.

Educational status of women was found to be the most significant factor influencing place of delivery. Women with secondary or higher level of education are more likely to utilize health facility delivery care services. This finding is consistent with previous studies which reported educational status of women to be the most significant associated factors for utilization of institutional delivery service [6]. This might be explained that a higher level of female education in the community leads to better awareness of the need for care during childbirth and female autonomy to utilize health facility services.

Several studies have revealed that antenatal care service utilization is a strong determinant of utilization of institutional delivery. Consistent with other study findings [9, 15-17], this study found that making one or more ANC visits is an important factor influencing delivery care service utilization. This might be advice from healthcare workers during antenatal care increases a woman's use of institutional delivery. The healthcare workers could provide good information regarding safe health care delivery and encourage women to deliver at health facility. A previous study in Ethiopia found that proper counseling and advice to deliver at health care facility increased institutional delivery [18]. This matter was also reflected from FGD participants: "During my last few days before I delivered, I went to the ANC clinic, and the midwife advised me sympathetically that I had to go to the district hospital for delivery because my child was not in the right position. I went to the district hospital and gave birth to healthy baby". (FGD participant, aged 32 years).

Another FGD participant shared her testimony about benefits of ANC attendance as stated beneath: "Benefits of ANC are well-recognized in our community. For example, pregnant women who had a good knowledge and attended ANC clinic usually delivers at health institution. In addition, women attend health institution for delivery after they encountered complications such as too much bleeding and/or prolonged labor". (A 35- year old FGD participant). In our study, women and their families thought that skilled birth care is necessary only when complications occur. A qualitative study among rural women in central Nepal shows similar results that women go to the health facility only if they experience a problem during labor [19].

Stable maternal source of income was also significantly associated with institutional delivery in this study. In principle, health care in Ethiopia for pregnant women is free. However, healthcare facilities seldom run out of stock, requiring a pregnant woman to incur some expenses for supplies or services. Besides, some women must pay transportation fare to travel from home to the health facility. Therefore, pregnant women with good sources of income are better able to pay for transport and unexpected healthcare facility costs. A previous study from Ethiopia also found that women with stable income were more likely to utilize institutional delivery [20].

The present study was correspondingly consistent with the studies conducted in the Latin American and Caribbean region, and in Europe, North Africa, and the Middle East, over $50 \%$ of all women in richest quintile reported that choosing delivery at public facilities [21].

Users of health care services often consider attitude of health workers when deciding where to seek care. Women often mentioned positive attitude of health workers as a main boosting factor for the utilization of health services particularly institutional delivery. The women reported varied witnesses. For example in the present study, a mother who had given birth to her third baby a month ago, gave a clear picture of a providerclient relationship: "When I went to the health facility for delivery, I was impressed by the nurse who cared for me so much. She was so human, respectful and compassionate" (FGD Participant, aged 28 years).

Expectations were commonly ruled by experience of the women which predisposed their future expectations. Women expect staff with a positive attitude. These included advice, politeness, giving reassurance, accessible by the bedside and exercising patience. Support, care 
and companionship throughout labor and delivery are exceedingly valued. Regrettably these were not always obtained in health facilities. As these were always assured for home deliveries women opt to deliver at home as oppose to a health facility [21].

Procedures during labor can be started with little discussion, but might be considered disgraceful or awful to women. For instance, vaginal examinations women are exposed to in labor and delivery is frequently regarded and stated as painful, frequent and often cited as a factor deterring the use of health facility for delivery. A woman who had her $2^{\text {nd }}$ child in the hospital narrated: "in $m y$ previous delivery I was exposed to series of vaginal examination....... Had it been my first delivery with the health facility, then I would not ever go there again". My faith (Muslim) doesn't let another person to insert his hands into a married woman's genital parts (Vagina). (FGD Participant, 34 years).

The midwife should be able to perform vaginal examination; ensuring the woman's own well-being. The required skills and ability of a midwife includes being able to communicate commendably, need to foster effective interpersonal communication skills and an attitude of respect for the women's right to be a full partner in the management of their pregnancy, child birth and the post natal period [21].

There are several potential limitations to our study. First, as in all cross-sectional studies, we can infer association but not causation from our results. Second, social desirability bias could have magnified there ported rates of health facility delivery; nevertheless, the study was confidential and data collectors were instructed to guarantee women that their responses could not be related to them. Data on child birth and place of delivery were collected retrospectively from mothers so there is potential for recall bias; to minimize this, we used data on the most recent birth within the past 1 year. Third, our study was limited to 9 villages (1 urban and 8 rural) in one district and hence the findings cannot be generalized to the country or the region as a whole.

\section{Conclusion}

This study revealed that women's institutional delivery service utilization in the study area is low. We identified significant factors that determine utilization of institutional delivery care in rural Ethiopia. Factors promoting the choice of institutional delivery were education level, ANC attendance, and high family income. The qualitative data has described and gave an insight of the contributing factors that influence the women using the health institutions for delivery. These included: ANC attendance, positive attitude of health workers and complications during labor and delivery. The preference for a health facility delivery was largely due to the understanding that if complications occurred either during labor or delivery, this was the only place where they could be managed.

We thus recommend that the government takes the matter of female education and empowerment more earnestly besides to steady health education on the hazard of home delivering as a method of decreasing maternal mortality in the country and achieving the millennium development goals 5. Maternal health care utilization would be fortified by making services more accessible, providing training for health care personnel, mainly health extension workers in health posts, and adopting a strategy to make the services available when needed. Services friendly to women should also be encouraged in all health facilities that provide maternal health care.

\section{Acknowledgments}

We are grateful to Addis Ababa University, College of Health Sciences, School of Allied Health, for its financial and technical support. We are also very indebted to extend our gratitude to West Gojjam Health Bureau, Daga Damot District health bureau and the District administration office for their kind assistance during the entire process of the study. The authors are also thankful to the respondents who offered their time to participate in this study. Special thanks go to the research assistants who participated in data collection.

\section{Funding}

Funding for this study was made possible through grants offered by Addis Ababa University post graduate office.

\section{Authors' contributions}

AS conceptualized the paper, searched literature, trained field researchers for data collection and wrote the results and discussion sections. He also wrote the manuscript draft. EG contributed to the design of the study and provided advice regarding methods, data interpretation and analyzed the qualitative data. He also critically reviewed and edited the manuscript. Both authors read and approved the final manuscript.

\section{Competing interests}

The authors declare that they have no competing interests.

\section{Consent for publication}

Not applicable for this study.

\section{Ethics approval and consent to participate}

The study secured ethical clearance from the Institutional Review Board (IRB) of Addis Ababa University. Authorized letter was obtained from Dega Damot Health District Administration Office to conduct the study. Study participants were informed about the purpose of the study, their right to refuse or withdraw from the study. Confidentiality was ensured by excluding their names or address in the questionnaire and final report. There were no psychological or social risks or discomforts involved in participating in this study.

Participants were informed through a written informed consent that participation was voluntary and that she had the option to stay out of the study at any point and time during the study. All questionnaires were put in a well labeled sealed large envelope and kept in a metal cabinet under lock and key.

\section{Author details}

'Department of Nursing, Mizan Tepi University, College of Health Sciences, Mizan Teferi, Ethiopia. ${ }^{2}$ Department of Nursing and Midwifery, Addis Ababa University, College of Health Sciences, Addis Ababa, Ethiopia, P.O. Box 1176, Ethiopia. 
Received: 6 February 2015 Accepted: 8 August 2016

\section{Published online: 17 August 2016}

\section{References}

1. United States agency for international development (USAID). 2014. Ending preventable maternal mortality: USAID Maternal Health Vision for action. USAID. https://www.evolve.elservier.com.

2. United Nations (UN). The millennium development goals. New York: United Nations; 2011.

3. World Health Organization. World Health Statistics. Switzerland: World Health Organization; 2012. Available from: http://www.who.int/gho/ publications/world_health_statistics/2012/en/. [Accessed 17 Nov 2015].

4. United Nations (UN). The millennium development goals report. New York: United Nations; 2015.

5. Central Statistical Agency [Ethiopia]. Ethiopia mini demographic and health survey. Addis Ababa; 2014. https://www.ethiopia/Mini_DHS_2014_Final_ Report.pdf. Accessed 3 Oct 2015.

6. Central Statistical Agency [Ethiopia] and ICF International. Ethiopia Demographic and Health Survey 2011: Addis Ababa. Ethiopia and Calverton: Central Statistical Agency and ICF International; 2012.

7. Berhan Y, Berhan A. Antenatal care as a means of increasing birth in the health facility: A systematic Review. Ethiop J Health Sci. Special issue. 2014;24:8-10. http://dx.doi.org/10.4314/ejhs.v24i1.9S. Accessed 15 Apr 2015

8. CSA. Amhara - Ethio Demography and Health. 2007. [accessed on 8 Jan 2014]; Available from: http://www.ethiodemographyandhealth.org/Amhara.html.

9. Teferra AS, Alemu FM, Woldeyohannes SM. Institutional delivery service utilization and associated factors among mothers who gave birth in the last 12 months in Sekela District, North West of Ethiopia: a community-based cross sectional study. BMC Pregnancy Childbirth. 2012;12(1):74.

10. Agnes A, David M, George BO, Fred N. Predictors for health facility delivery in Busia district of Uganda: a cross sectional study. BMC Pregnancy Childbirth. 2012;12:132.

11. PubMed / Google Scholar, Nair M, Ariana P, Webster P. What influences the decision to undergo institutional delivery by skilled birth attendants? A cohort study in rural Andhra Pradesh, India. Rural Remote Health. 2012;12: 2311. PubMed | Google Scholar.

12. FMOH. Health Sector Development Programme IV (HSDP IV) 2010/11-2014/ 15. Addis Ababa: FMOH; 2010.

13. Kesterton AJ, Cleland J, Sloggett A, Ronsmans C. Institutional Delivery in Rural India: the relative importance of accessibility and economic status. BMC Pregnancy Childbirth. 2010;10(30).

14. Garg R, Shyamsunder D, Singh T, Singh PA. Study on delivery practices among Women in Rural Punjab. Health Popul. 2010;33(1):23-33.

15. Pfeiffer C, Mwaipopo R. Delivering at home or in a health facility? Healthseeking behavior of women and the role of traditional birth attendants in Tanzania. BMC Pregnancy Childbirth. 2013;13(1):55.

16. Singh S, Darroch JE. Adding it up: costs and benefits of contraceptive services. Guttmacher Institute and UNFPA; 2012. https://www.evolve. elservier.com

17. Rose NM M, Killewo JZ, Leshabari MT, Massawe SN, Albrecht J, Declare M, Hassan M. Use pattern of maternal health services and determinants of skilled care during delivery in Southern Tanzania: implications for achievement of MDG-5 targets. BMC Pregnancy Childbirth. 2007;7:29.

18. Yalem T, Tesfay G, Isabel G, Kerstin E, Haile Mariam L, Migue SS. Determinants of antenatal and deliveries care utilization in Tigray region, Ethiopia: a cross-sectional study. Int J Equity Health. 2013;12:30.

19. Morrison J, Thapa R, Basnet M, Budhathoki B, Tumbahangphe K, Manandhar $D$, et al. Exploring the first delay: a qualitative study of home deliveries in Makwanpur District, Nepal. BMC Pregnancy Childbirth. 2014;14:89.

20. Alkema L, New JR, Pedersen J, You D. Child mortality estimation 2013: an overview of updates in estimation methods by the United Nations InterAgency Group for Child Mortality Estimation. 2014.

21. Francis Abugri AKUM. A qualitative study on factors contributing to low institutional child delivery rates in Northern Ghana: the case of Bawku Municipality. J Community Med Health Educ. 2013;(3):236. doi:10.4172/21610711.1000236

\section{Submit your next manuscript to BioMed Central and we will help you at every step:}

- We accept pre-submission inquiries

- Our selector tool helps you to find the most relevant journal

- We provide round the clock customer support

- Convenient online submission

- Thorough peer review

- Inclusion in PubMed and all major indexing services

- Maximum visibility for your research

Submit your manuscript at www.biomedcentral.com/submit 